\title{
HIGHER DERIVATIONS AND CENTRAL SIMPLE ALGEBRAS
}

\author{
A. ROY and R. SRIDHARAN \\ (Dedicated to the memory of Tadasi Nakayama)
}

Introduction. Let $K$ be a commutative ring, $A$ a $K$-algebra, and $B$ a $K$-subalgebra of $A$. The object of this paper is to prove some results on higher derivations (in the sense of Jacobson [4]) of $B$ into $A$. In $\S 1$ we introduce a notion of equivalence among higher derivations. With this notion of equivalence, we prove in $\$ 2$ (Theorem 1) that the equivalence classes of higher $K$-derivations of $B$ into $A$ are in one-one correspondence with the isomorphism classes of certain filtered $B \otimes{ }_{K} A^{\circ}$-modules, where $A^{\circ}$ denotes the opposite algebra of $A$. In $\$ 3$ we give a cohomological criterion for the extendability of a higher derivation of a commutative ring to a crossed product. We use this result in $\$ 4$ to show (Theorem 2) that if $A$ is central simple over $K$ and $B$ is semi-simple, then any higher derivation of $B$ into $A$ which maps $K$ into $K$ can be extended to a higher derivation of $A$. This result is a generalization of a theorem of JacobsonHochschild ([2], Theorem 6) on extendability of derivations.

\section{\&1 Generalities on higher derivations.}

Let $B$ be a subring of a ring $A$. We recall that a higher derivation of rank $n$ of $B$ into $A$ is a sequence of additive maps $\delta=\left(d_{0}=1, d_{1}, \cdots, d_{n}\right)$ of $B$ into $A$ such that

$$
d_{i}\left(b b^{\prime}\right)=\sum_{0 \leqslant j \leqslant i} d_{j}(b) d_{i-j}\left(b^{\prime}\right),
$$

$b, b^{\prime} \in B, 0 \leqslant i \leqslant n$. If $A$ is an algebra over a commutative ring $K$ and $B$ a $K$-subalgebra of $A$, then $\delta$ is called a higher $K$-derivation if the maps $d_{i}$ are $K$-linear, i.e. if the maps $d_{i}$ vanish on $K$ for $i \geqslant 1$. The following statement is easily checked:

$$
\text { If }\left(d_{0}=1, d_{1}, \cdots, d_{n-1}, d_{n}\right) \text { and }\left(d_{0}=1, d_{1}, \cdots, d_{n-1}, d_{n}^{\prime}\right)
$$

Received February 15, 1967. 
are higher derivations of $B$ into $A$, then $d_{n}-d_{n}^{\prime}$ is a derivation.

For any ring $\Lambda$, let $T_{n}(\Lambda)$ be the ring $\Lambda[X] /\left(X^{n+1}\right)$. We shall denote the image of $X$ in $T_{n}(\Lambda)$ by $x$. Let $\eta_{\Lambda}: T_{n}(\Lambda) \rightarrow \Lambda$ be the ring epimorphism defined by $\eta_{A}\left(\lambda_{0}+\lambda_{1} x+\cdots+\lambda_{n} x^{n}\right)=\lambda_{0}$. Since ker $\eta_{A}$ is nilpotent, $1+$ ker $\eta_{A}$ is a subgroup of the group of units of $T_{n}(\Lambda)$. We shall denote this subgroup by $U_{n}(\Lambda)$.

With $A$ and $B$ as above, if $\delta: B \rightarrow A$ is a higher derivation, then the map $\alpha_{\delta}: B \rightarrow T_{n}(A)$ given by $\alpha_{\delta}(b)=\sum_{0 \leqslant i \leqslant n} d_{\imath}(b) x^{i}$ is a section of $\eta_{A}$ on $B$, i.e., $\alpha_{\delta}$ is a ring homomorphism such that $\eta_{A} \circ \alpha_{\delta}=$ identity. Conversely, let $\alpha$ be a section of $\eta_{A}$ on $B$. If $\alpha(b)=\sum_{0 \leqslant i \leqslant n} d_{i}(b) x^{i}$, then $\left(d_{0}=1, d_{1}, \cdots, d_{n}\right)$ is a higher derivation of $B$ into $A$.

If $\delta, \delta^{\prime}: B \rightarrow A$ are two higher derivations, we say that they are equivalent, if there exists an element $u \in U_{n}(A)$ such that $\alpha_{\delta^{\prime}}=$ int $u \circ \alpha_{\delta}$, where int $u$ denotes the inner automorphism of $T_{n}(A)$ given by $u$. Clearly, this is an equivalence relation. More explicitly, $\delta$ and $\delta^{\prime}$ are equivalent if and only if there exist elements $u_{0}=1, u_{1}, \cdots, u_{n} \in A$ such that

$$
\sum_{0 \leqslant j \leqslant i} u_{j} d_{i-j}(b)=\sum_{0 \leqslant j \leqslant i} d_{i-j}^{\prime}(b) u_{j}
$$

for $b \in B$ and $0 \leqslant i \leqslant n$. A higher derivation is called inner if it is equivalent to the higher derivation $\left(d_{0}=1, d_{1}, \cdots, d_{n}\right)$, where $d_{i}=0$ for $i \geqslant 1$.

\section{§ 2. Higher derivations and filtered modules}

Let $K$ be a commutative ring, $A$ a $K$-algebra, and $B$ a $K$-subalgebra of $A$. For any positive integer $n$, we denote by $\bar{A}(n)$, the graded $B \otimes{ }_{K} A^{\circ}$-module $\sum_{0 \leqslant i \leqslant n} \bar{A}_{i}$, where $\bar{A}_{i}$ is the $B \otimes{ }_{K} A^{\circ}$-module $A$. Let $\bar{e}_{i}$ denote the element 1 of $\bar{A}_{i}$. Let $\bar{\theta}$ denote the graded endomorphism of degree -1 of $\bar{A}(n)$ defined by $\bar{\theta}_{\imath}\left(\overline{\boldsymbol{e}}_{\imath}\right)=\overline{\boldsymbol{e}}_{\imath-1}$ for $i>0$, and $\bar{\theta}_{0}=0$.

We consider the class $\mathscr{C}$ of triples $(M, \psi, \theta)$, where $M$ is a $B \otimes_{K} A^{\circ}$ module with a filtration $0 \subset M_{0} \subset M_{1} \subset \cdots \subset M_{n}=M, \quad \theta$ a $B \otimes \otimes_{K} A^{\circ}$ endomorphism of degree -1 of $M$ and $\psi: E^{\circ}(M) \rightarrow \bar{A}(n)$ an isomorphism of graded $B \otimes{ }_{K} A^{\circ}$-modules, where $E^{\circ}(M)$ denotes the associated graded module of $M$, such that the diagram 


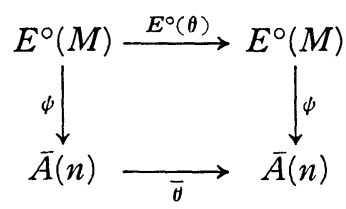

is commutative. With the natural filtration on $\bar{A}(n)$, the triple $\left(\bar{A}(n), 1_{\bar{A}(n)}, \bar{\theta}\right)$ is clearly a member of $\mathscr{C}$. We define a morphism $(M, \psi, \theta) \rightarrow$ $\left(M^{\prime}, \psi^{\prime}, \theta^{\prime}\right)$ in $\mathscr{C}$ to be a map of filtered $B \otimes{ }_{K} A^{\circ}$-modules $M \rightarrow M^{\prime}$ which is compatible with $\psi, \psi^{\prime}$ and $\theta, \theta^{\prime}$.

Thus $\mathscr{C}$ becomes a category. Clearly, every morphism in $\mathscr{C}$ is an isomorphism.

Let $\delta=\left(d_{0}=1, d_{1}, \cdots, d_{n}\right)$ be a higher $K$-derivation of rank $n$ of $B$ into $A$. On the free right $A$-module $A_{\delta}=\sum_{0 \leqslant i \leqslant n} e_{i} A$, with basis $\left(e_{\imath}\right)$, we define a left $B$-module structure by setting $b\left(e_{i} a\right)=\left(\sum_{0 \leqslant j \leqslant i} e_{j} d_{i-j} b\right) a$ for $0 \leqslant i \leqslant n, b \in B, a \in A$. This makes $A_{\delta}$ a $B \otimes{ }_{K} A^{\circ}$-module. We define a filtration $0 \subset\left(A_{\hat{\delta}}\right)_{0} \subset\left(A_{\hat{\delta}}\right)_{1} \subset \cdots \subset\left(A_{\hat{\delta}}\right)_{n}=A_{\delta}$ by taking $\left(A_{\hat{\delta}}\right)_{i}$ to be the $B \otimes_{K} A^{\circ}$-submodule of $A_{\delta}$ generated by $e_{0}, \cdots, e_{i}$. We also define a $B \otimes{ }_{K} A^{\circ}$-endomorphism $\theta_{\delta}$ of degree -1 of the filtered module $A_{\delta}$ by setting $\theta_{\delta}\left(e_{0}\right)=0$ and $\theta_{\delta}\left(e_{i}\right)=e_{i-1}$ for $i \geqslant 1$. The map $\left(A_{\delta}\right)_{i} \rightarrow \bar{A}_{i}$ which sends $\sum_{0 \leqslant j \leqslant i} e_{j} a_{j}$ to $\bar{e}_{i} a_{i}$ is $B \otimes{ }_{K} A^{\circ}$-linear. This map is an isomorphism for $i=0$ and has $\left(A_{\delta}\right)_{i-1}$ as its kernel for $i \geqslant 1$. We thus get an isomorphism

$$
\psi_{\delta}: E^{\circ}\left(A_{\delta}\right) \rightarrow \bar{A}(n)
$$

of graded $B \otimes_{K} A^{\circ}$-modules. Clearly, $\left(A_{\delta}, \psi_{\delta}, \theta_{\delta}\right)$ is an object of $\mathscr{C}$.

Now let $\delta=\left(d_{0}=1, d_{1}, \cdots, d_{n}\right)$ and $\delta^{\prime}=\left(d_{0}^{\prime}=1, d_{1}^{\prime}, \cdots, d_{n}^{\prime}\right)$ be two equivalent higher $K$-derivations of $B$ into $A$. There exist elements $u_{0}=1, u_{1}, \cdots, u_{n} \in A$ satisfying the condition (*) of $\S 1$. The isomorphism $A_{\delta} \rightarrow A_{\delta^{\prime}}$ of right $A$-modules which sends $e_{i}$ to $\sum_{0 \leqslant j \leqslant i} e_{j}^{\prime} u_{i-j}$ is easily verified to be left $B$-linear and actually gives an isomorphism in $\mathscr{C}$ of $\left(A_{\delta}, \psi_{\delta}, \theta_{\delta}\right)$ onto $\left(A_{\dot{\partial}^{\prime}}, \psi_{\dot{\delta}^{\prime}}, \theta_{\partial^{\prime}}\right)$. Thus, equivalent higher $K$-derivations of $B$ into $A$ give rise to isomorphic objects in $\mathscr{C}$.

Consider now any object $(M, \psi, \theta) \in \mathscr{C}$. We then have for $1 \leqslant i \leqslant n$, the following commutative diagrams with exact rows: 


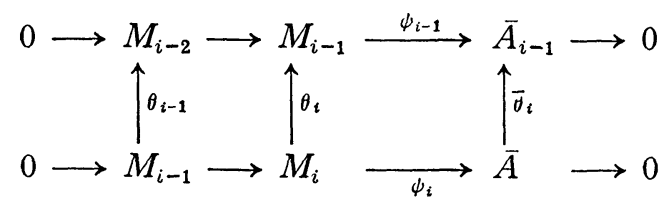

where $M_{-1}=0$. Let $s_{n}: \bar{A}_{n} \rightarrow M_{n}$ be a right $A$-linear map such that $\psi_{n} \circ s_{n}=$ identity. The map $s_{n}$ induces right $A$-linear maps $s_{i}(0 \leqslant i<n)$ such that $\theta_{i} \circ s_{i}=s_{i-1} \circ \bar{\theta}_{i}$ and we have $\psi_{i} \circ s_{i}=$ identity. If $s_{i}\left(\bar{e}_{i}\right)=m_{i}$, we have $M_{i}=m_{0} A+m_{1} A+\cdots+m_{\imath} A$. Since for any $b \in B, \psi_{i}\left(b m_{i}-m_{i} b\right)=0$, it follows that $b m_{i}-m_{\imath} b \in M_{i-1}$. Let $b m_{n}-m_{n} b=\sum_{0 \leqslant i \leqslant n-1} m_{i} d_{n-i} b$. Applying $\theta_{i+1} \circ \cdots \circ \theta_{n}$, we get

$$
b m_{i}-m_{i} b=\sum_{0 \leqslant j \leqslant i-1} m_{j} d_{2-j} b,
$$

since $\theta_{i}\left(m_{j}\right)=m_{j-1}$ for $1 \leqslant j \leqslant i$ and $\theta_{i}\left(m_{0}\right)=0$. Now (setting $d_{0}=1$ )

$$
\begin{gathered}
\sum_{0 \leqslant k \leqslant n-1} m_{n-k} d_{k}\left(b b^{\prime}\right)=b b^{\prime} m_{n}-m_{n} b b^{\prime} \\
=b\left(b^{\prime} m_{n}-m_{n} b^{\prime}\right)+\left(b m_{n}-m_{n} b\right) b^{\prime} \\
=\sum_{0 \leqslant i \leqslant n-1} b m_{i} d_{n-i} b^{\prime}+\left(\sum_{0 \leqslant i \leqslant n-1} m_{i} d_{n-i} b\right) b^{\prime} \\
=\sum_{0 \leqslant i \leqslant n-1}\left(\sum_{0 \leqslant j \leqslant i} m_{j} d_{i-j} b\right) d_{n-i} b^{\prime} \\
\quad+\sum_{0 \leqslant i \leqslant n-1} m_{i}\left(d_{n-i} b\right) b^{\prime} .
\end{gathered}
$$

Comparing the coefficients of $m_{n-k}$ on both sides, we get

$$
d_{k}\left(b b^{\prime}\right)=\sum_{0 \leqslant i \leqslant k} d_{i}(b) d_{k-i}\left(b^{\prime}\right), \quad 1 \leqslant k \leqslant n,
$$

i.e. $\delta=\left(d_{0}=1, d_{1}, \cdots, d_{n}\right)$ is a higher derivation of rank $n$ of $B$ into $A$.

The right $A$-linear map $f: A_{\delta} \rightarrow M$ defined by $f\left(e_{i}\right)=m_{i}$ is clearly $B$-linear, and is in fact an isomorphism in $\mathscr{C}$.

Let now $s_{n}^{\prime}: \bar{A}_{n} \rightarrow M_{n}$ be another right $A$-linear map such that $\psi_{n} \circ s_{n}^{\prime}$ $=$ identity and let $s_{i}^{\prime}: \bar{A}_{i} \rightarrow M_{i}$ be such that $\theta_{i} \circ s_{i}^{\prime}=s_{i-1}^{\prime} \circ \bar{\theta}_{i}$ for $0 \leqslant i \leqslant n$. Let $s_{i}^{\prime}\left(\bar{e}_{i}\right)=m_{i}^{\prime}$. Since $\psi_{n}\left(m_{n}^{\prime}-m_{n}\right)=0$, we have $\dot{m}_{n}^{\prime}-m_{n} \in M_{n-1}$. We thus have elements $u_{0}=1, u_{1}, \cdots, u_{n} \in A$ such that

$$
m_{n}^{\prime}=\sum_{0 \leqslant i \leqslant n} m_{n-i} u_{i}
$$

Applying $\theta_{k+1} \circ \cdots \circ \theta_{n}$, we get 


$$
m_{k}^{\prime}=\sum_{0 \leqslant i \leqslant k} m_{k-i} u_{i}
$$

Let $\delta^{\prime}=\left(d_{0}^{\prime}=1, d_{1}^{\prime}, \cdots, d_{n}^{\prime}\right)$ be the higher $K$-derivation corresponding to $s_{n}^{\prime}$. Then, for any $b \in B$,

$$
b m_{k}^{\prime}-m_{k}^{\prime} b=\sum_{1 \leqslant i \leqslant k} m_{k-i}^{\prime} d_{i}^{\prime} b .
$$

From $(*)_{n}$ we have,

$$
\begin{aligned}
\sum_{1 \leqslant i \leqslant n} m_{n-i}^{\prime} d_{i}^{\prime} b & =b m_{n b}^{\prime}-m_{n}^{\prime} b \\
& =\sum_{0 \leqslant i \leqslant n} b m_{n-i} u_{i}-\sum_{0 \leqslant i \leqslant n} m_{n-i} u_{i} b \\
& =\sum_{0 \leqslant i \leqslant n}\left(\sum_{0 \leqslant j \leqslant n-i} m_{j} d_{n-i-j} b\right) u_{\imath}-\sum_{0 \leqslant i \leqslant n} m_{n-\imath} u_{\imath} b .
\end{aligned}
$$

Substituting for $m_{n-i}^{\prime}$ from $(*)_{n-i}$ in the above equation, and comparing the coefficients of $m_{n-k}$, we get

$$
\sum_{0 \leqslant i \leqslant k} u_{i} d_{k-i}^{\prime} b=\sum_{0 \leqslant i \leqslant k} d_{k-i} b u_{\imath}
$$

for $0 \leqslant k \leqslant n$. Thus $\delta^{\prime}$ is equivalent to $\delta$. It follows now that for a given isomorphism class in $\mathscr{C}$, there exists a higher $K$-derivation $\delta$ of $B$ into $A$, unique up to equivalence, such that $\left(A_{\delta}, \psi_{\delta}, \theta_{\delta}\right)$ belongs to that class.

Thus we have the following

Theorem 1. Let $A$ be a K-algebra, $B$ a K-subalgebra, and let $\mathscr{C}$ denote the category of triples $(M, \psi, \theta)$ constructed above. The map $\delta /\left(A_{\delta}, \psi_{\delta}, \theta_{\delta}\right)$ of the set of higher $K$-derivations $\delta: B \rightarrow A$ into obj $\mathscr{C}$ induces a bijection of the set of equivalence classes of these higher derivations onto the set of isomorphism classes of obj $\mathscr{C}$. Under this bijection, the equivalence class of inner higher derivations corresponds to the isomorphism class of $\left(\bar{A}(n), 1_{\bar{A}(n)}, \bar{\theta}\right)$.

\section{§ 3. Extension of higher derivations to crossed products.}

Let $L$ be a commutative ring and let $\delta: L \rightarrow L$ be a higher derivation of rank $n$. Let $L^{*}$ denote the group of units of $L$. We then have a homomorphism $\delta^{*}: L^{*} \rightarrow U_{n}(L)$ of groups, defined by

$$
\delta^{*}(\lambda)=\sum_{0 \leqslant i \leqslant n} \lambda^{-1} d_{i} \lambda x^{i}, \quad \lambda \in L^{*}
$$

Now, let $G$ be a finite group of automorphisms of $L$. Let $G$ operate 
on $T_{n}(L)$ by setting $s \sum \lambda_{i} x^{i}=\sum s\left(\lambda_{i}\right) x^{i}, s \in G, \lambda_{i} \in L$. Clearly $U_{n}(L)$ is stable under the action of $G$. If $\delta$ is a higher $G$-derivation (i.e., if $d_{i}$ os $=\operatorname{sod}_{i}$ for all $s \in G$ and $\left.0 \leqslant i \leqslant n\right)$, then $\delta^{*}$ is a $G$-homomorphism. Thus $\delta^{*}$ induces a homomorphism $H^{2}\left(\delta^{*}\right): H^{2}\left(G, L^{*}\right) \rightarrow H^{2}\left(G, U_{n}(L)\right)$. Let $f$ : $G \times G \rightarrow L^{*}$ be a 2-cocycle. We recall that the crossed product $(L, G, f)$ is defined to be the free left $L$-module with a basis $\left(e_{s}\right)_{s \in G}$ together with a multiplication given by $\left(\lambda e_{s}\right)\left(\mu e_{t}\right)=\lambda s(\mu) f(s, t) e_{s t}, \lambda, \mu \in L, s, t \in G$.

Proposition 1. A higher $G$-derivation $\delta: L \rightarrow L$ can be extended to a higher derivation of the crossed product $A=(L, G, f)$ if $H^{2}\left(\delta^{*}\right)(\bar{f})=0$, where $\bar{f}$ denotes the class of $f$. Conversely, if $L$ is an integral domain and $\delta$ admits of an extension to $A$, then $H^{2}\left(\delta^{*}\right)(\bar{f})=0$.

Proof. Let $H^{2}\left(\delta^{*}\right)(\bar{f})=0$. This means that there exists a map $h: G \rightarrow$ $U_{n}(L)$ such that

$$
\delta^{*} f(s, t) h(s t)=h(s) \operatorname{sh}(t), \quad s, t \in G .
$$

Let $h(s)=\sum h_{i}(s) x^{i}$. We define additive maps $\bar{d}_{i}: A \rightarrow A$ by setting

$$
\bar{d}_{i}\left(\lambda e_{s}\right)=\sum_{0 \leqslant j \leqslant i} d_{j}(\lambda) h_{i-j}(s) e_{s}, \lambda \in L, s \in G .
$$

It is straightforward to check that $\left(\bar{d}_{0}=1, \bar{d}_{1}, \cdots, \bar{d}_{n}\right)$ is a higher derivation of $A$ which extends $\delta$.

Suppose now that $L$ is an integral domain and that $\bar{\delta}=\left(\bar{d}_{0}\right.$ $\left.=1, \bar{d}_{1}, \cdots, \bar{d}_{\mathfrak{n}}\right)$ is an extension of $\delta$ to $A$. We first show that for any $i(0 \leqslant \imath \leqslant n)$, we have $\bar{d}_{i}\left(e_{s}\right)=h_{\imath}(s) e_{s}$ for some map $h_{i}: G \rightarrow L$. For, let this be assumed proved for $0 \leqslant j<i$ and let $\bar{d}_{i}\left(e_{s}\right)=\sum_{t \in G} h_{i}(s, t) e_{t} ; h_{i}(s, t) \in L$. For any $\lambda \in L$, we have

$$
\begin{aligned}
\bar{d}_{i}\left(e_{s} \lambda\right) & =\sum_{0 \leqslant j \leqslant i}\left(\bar{d}_{i-j} e_{s}\right)\left(d_{j} \lambda\right) \\
& =\sum_{t \in G}\left(h_{i}(s, t) e_{t}\right) \lambda+\sum_{1 \leqslant j \leqslant i}\left(h_{i-j}(s) e_{s}\right) d_{j}(\lambda) .
\end{aligned}
$$

On the other hand,

$$
\begin{aligned}
\bar{d}_{i}\left(e_{s} \lambda\right) & =\bar{d}_{i}\left(s(\lambda) e_{s}\right)=\sum_{0 \leqslant j \leqslant i} d_{j} s(\lambda) \bar{d}_{i-j} e_{s} \\
& =\sum_{1 \leqslant j \leqslant i} d_{j} s(\lambda) h_{i-j}(s) e_{s}+s(\lambda) \sum_{t \in G} h_{i}(s, t) e_{t} .
\end{aligned}
$$

Comparing the coefficients of $e_{t}$ for $t \neq s$, we get 


$$
h_{i}(s, t) t(\lambda)=h_{i}(s, t) s(\lambda),
$$

for all $\lambda \in L$. Since $t(\lambda) \neq s(\lambda)$ for some $\lambda$, it follows that $h_{i}(s, t)=0$ for $s \neq t$. Thus we have functions $h_{i}: G \rightarrow L$ such that $\bar{d}_{i}\left(e_{s}\right)=h_{i}(s) e_{s}$, $0 \leqslant i \leqslant n$.

Now

$$
\begin{aligned}
\bar{d}_{i}\left(e_{s} e_{t}\right) & =\sum_{0 \leqslant j \leqslant i}\left(\bar{d}_{j} e_{s}\right)\left(\bar{d}_{i-j} e_{t}\right) \\
& =\sum_{0 \leqslant j \leqslant i} h_{j}(s) s h_{i-j}(t) f(s, t) e_{s t} .
\end{aligned}
$$

On the other hand

$$
\begin{aligned}
\bar{d}_{i}\left(e_{s} e_{t}\right) & =\bar{d}_{i}\left(f(s, t) e_{s t}\right) \\
& =\sum_{0 \leqslant j<i} d_{j} f(s, t) h_{i-j}(s t) e_{s t} .
\end{aligned}
$$

Thus, we have, for every $i$,

$$
\sum_{0 \leqslant j \leqslant i} d_{j} f(s, t) h_{i-j}(s t)=\sum_{0 \leqslant j \leqslant i} h_{j}(s) s h_{i-j}(t) .
$$

If $h: G \rightarrow U_{n}(L)$ is defined by $h(s)=\sum_{0 \leqslant i \leqslant n} h_{i}(s) x^{i}$, then the above equations can be written as

$$
\delta^{*} f(s, t) h(s t)=h(s) \operatorname{sh}(t),
$$

which shows that $H^{2}\left(\delta^{*}\right)(\bar{f})=0$.

Corollary. If $H^{2}(G, L)=0$, then any higher $G$-derivation of $L$ can be extended to any crossed product of $G$ and $L$.

The above corollary is an immediate consequence of the above proposition and the following

Lemma. If $H^{2}(G, L)=0$, then $H^{2}\left(G, U_{n}(L)\right)=0$ for every $n$.

Proof. We define a $G$-homomorphism $L \rightarrow U_{n}(L)$ by mapping $\lambda$ into $1+\lambda x^{n}$. This is an isomorphism for $n=1$ and so $H^{2}\left(G, U_{1}(L)\right)=0$. For $n>1$ we have an exact sequence of $G$-modules

$$
0 \rightarrow L \rightarrow U_{n}(L) \rightarrow U_{n-1}(L) \rightarrow 1
$$

where the map $U_{n}(L) \rightarrow U_{n-1}(L)$ sends $\sum_{0 \leqslant i \leqslant n} \lambda_{i} x^{i}$ to $\sum_{0 \leqslant i \leqslant n-1} \lambda_{i} x^{i}$. We then have an exact sequence 


$$
H^{2}(G, L) \rightarrow H^{2}\left(G, U_{n}(L)\right) \rightarrow H^{2}\left(G, U_{n-1}(L)\right) .
$$

It follows by induction on $n$ that $H^{2}\left(G, U_{n}(L)\right)=0$.

\section{§4. Higher derivations and central simple algebras}

The aim of this section is to establish the following

Theorem 2. Let $A$ be a finite dimensional central simple K-algebra and let $B$ be a semi-simple subalgebra of $A$. Then any higher derivation of $B$ into $A$, which maps $K$ into itself, can be extended to a higher derivation of $A$.

Before proving the theorem, we prove a few lemmas.

Lemma 1. Let $A$ be a ring, $B$ a subring of $A$, and let $\delta, \delta^{\prime}: B \rightarrow A$ be two equivalenl higher derivations of rank $n$. If $\delta$ admits of an extension to $A$ then $\delta^{\prime}$ can also be extended to $A$ such that these extensions are equivalent.

Proof. Let $u \in U_{n}(A)$ be such that $\alpha_{\delta^{\prime}}=$ int $u \circ \alpha_{\delta}$. If $\bar{\delta}$ is an extension of $\delta$ to $A$, then int $u \circ \alpha_{\bar{\delta}}: A \rightarrow T_{n}(A)$ is a section of $\eta_{A}: T_{n}(A) \rightarrow A$ on $A$. This section gives the required extension of $\delta^{\prime}$ to $A$.

Lemma 2. Let $A$ be a K-algebra and let $B$ be a K-subalgebra of $A$ such that every K-derivation of $B$ into $A$ is inner. Let $\delta, \delta^{\prime}: B \rightarrow A$ be higher derivations. of rank $n$ mapping $K$ into itself such that $\delta / K=\delta^{\prime} / K$. Then $\delta$ and $\delta^{\prime}$ are equivalent.

Proof. The case $n=1$ follows from the hypothesis that the $K$-derivations of $B$ into $A$ are inner.

Let now $n>1$ and assume by induction that $\delta_{1}=\left(d_{0}=1, d_{1}, \cdots, d_{n-1}\right)$ and $\delta_{1}^{\prime}=\left(d_{0}^{\prime}=1, d_{1}^{\prime}, \cdots, d_{n-1}^{\prime}\right)$ are equivalent. Let $u=1+u_{1} x+\cdots$ $+u_{n-1} x^{n-1} \in U_{n-1}(A)$ be such that $\alpha_{\delta_{1}^{\prime}}=$ int $u \circ \alpha_{\delta_{1}}$. Consider the element $v=1+u_{1} x+\cdots+u_{n-1} x^{n-1} \in U_{n}(A)$. The homomorphism int $v \circ \alpha_{\delta}: B$ $\rightarrow T_{n}(A)$ gives a higher derivation $\delta^{\prime \prime}=\left(d_{0}^{\prime \prime}=1, d_{1}^{\prime \prime}, \cdots, d_{n}^{\prime \prime}\right)$ equivalent to $\delta$ such that $d_{i}^{\prime \prime}=d_{i}^{\prime}$ for $0 \leqslant i \leqslant n-1$. Further $d_{n}^{\prime \prime} / K=d_{n}^{\prime} / K$. Thus $d_{n}^{\prime \prime}-d_{n}^{\prime}$ is a $K$-derivation of $B$ into $A$. Therefore there exists a $u_{n} \in A$ such that $d_{n}^{\prime \prime}(b)-d_{n}^{\prime}(b)=u_{n} b-b u_{n}$. It is easily verified that $\alpha_{\delta^{\prime \prime}}=$ int (1 $\left.+u_{n} x^{n}\right) \circ \alpha_{\delta^{\prime}}$. Thus $\delta^{\prime \prime}$ and $\delta^{\prime}$ are equivalent, which proves the lemma.

Lemma 3. Let $K$ be a field and $L / K$ a finite separable extension. Then any higher derivation of $K$ into itself can be uniquely extended to a higher derivation of $L$. 
Proof. Let $L=K(\lambda)$ and let $f$ be the minimal polynomial of $\lambda$ so that we have an isomorphism $K[X] /(f) \rightarrow L$ under which $X$ goes to $\lambda$.

Let $\delta=\left(d_{0}=1, d_{1}, \cdots, d_{n}\right)$ be a higher derivation of $K$. We remark that $\delta$ can be extended to a higher derivation $\delta^{\prime}=\left(d_{0}^{\prime}=1, d_{1}^{\prime}, \cdots, d_{n}^{\prime}\right)$ of $K[X]$ by prescribing arbitrary values for $d_{1}^{\prime} X, \cdots, d_{n}^{\prime} X$.

Suppose, by induction, that $\left(d_{0}=1, d_{1}, \cdots, d_{n-1}\right)$ has been extended to a higher derivation $\left(d_{0}^{\prime}=1, d_{1}^{\prime}, \cdots, d_{n-1}^{\prime}\right)$ of $K[X]$ such that the ideal generated by $f(X)$ is stable under each $d_{i}^{\prime}$. Suppose further, that the induced higher derivation $\left(\bar{d}_{0}=1, \bar{d}_{1}, \cdots, \bar{d}_{n-1}\right)$ of $L$ is unique as an extension of $\left(d_{0}=1, d_{1}, \cdots, d_{n-1}\right)$.

Let $g$ be any element of $K[X]$. Let $\left(d_{0}^{\prime}=1, d_{1}^{\prime}, \cdots, d_{n}^{\prime}\right)$ be the higher derivation of $K[X]$ for which $d_{n}^{\prime} X=g$. It is easily seen that

$$
d_{n}^{\prime} f=f^{\prime} g+q,
$$

where $f^{\prime}$ is the usual derivative of $f$ and $q$ is a polynomial which depends only on $d_{1}^{\prime} X, \cdots, d_{n-1}^{\prime} X$. Since $f^{\prime}(\lambda) \neq 0$, there exists a polynomial $f_{1} \in K[X]$ such that $f_{1} f^{\prime} \equiv 1(\bmod f)$. If we choose $g=-f_{1} q$, then the ideal $(f)$ is stable under $d_{n}^{\prime}$, and the induced map $\bar{d}_{n}: L \rightarrow L$ satisfies $\bar{d}_{n}(\lambda)=-q(\lambda) / f^{\prime}(\lambda)$. Thus we have a higher derivation $\left(\bar{d}_{0}=1, \bar{d}_{1}, \cdots, \bar{d}_{n}\right)$ of $L$ which extends $\delta$ and is clearly unique.

Proof of Theorem 2. We first assume that the theorem is true with $B=K$ and prove it for the general case. Let $\delta$ be a higher derivation of $B$ into $A$ which maps $K$ into itself and let $\delta$ be an extension of $\delta / K$. The restrictions of $\delta$ and $\bar{\delta} / B$ to $K$ are the same. Since any $K$-derivation of $B$ into $A$ is inner ([3], Theorem 7), it follows from lemmas 1 and 2, that $\delta$ can be extended to $A$.

We now prove the theorem in the case $B=K$. Let $\delta$ be a higher derivation of $K$. We first show that it is enough to extend $\delta$ to some central simple $K$-algebra $A_{1}$ similar to $A$. In fact, let $\bar{\delta}$ be an extension of $\delta$ to $A_{1}$. If $D$ denotes the division algebra of $A_{1}$, we have $A_{1}=M_{m}(D)$ for some integer $m$. Let $\delta_{1}$ be the entrywise extension of $\delta$ to $M_{m}(K)$. Since $\delta_{1}$ and $\bar{\delta} / M_{m}(K)$ coincide on $K$ and since any $K$-derivation of $M_{m}(K)$ into $A_{1}$ is inner, it follows by lemmas 1 and 2 , that $\delta_{1}$ can be extended to a higher derivation $\bar{\delta}_{1}$ of $A_{1}$. Since $M_{m}(K)$ is stable under $\bar{\delta}_{1}$, and $D$ is the commutant of $M_{m}(K)$ in $A_{1}, D$ is also stable under $\bar{\delta}_{1}$. Thus, 
$\bar{\delta}_{1} / D$ is an extension of $\delta$, and this can be further extended to $A$, since $A$ is a matrix ring over $D$.

We can therefore assume that $A$ is a crossed product $(L, G, f)$ for some Galois extension $L / K$, where $G$ is the Galois group of $L / K$ ([1], Theorem 1, p. 66). By lemma 3 we have a unique extension $\bar{\delta}=\left(\bar{d}_{0}=1, \bar{d}_{1}, \cdots, \bar{d}_{n}\right)$ of $\delta$ to $L$. If $s \in G$, then $s \bar{\delta} s^{-1}=\left(s \bar{d}_{0} s^{-1}=1, s \bar{d}_{1} s^{-1}, \cdots, s \bar{d}_{n} s^{-1}\right)$ is also a higher derivation of $L$ extending $\delta$, so that we have $s \bar{d}_{i} s^{-1}=d_{i}$ for $0 \leqslant i \leqslant n$. In other words, $\bar{\delta}$ is a $G$-derivation. Since $H^{2}(G, L)=0$, it follows from the corollary to proposition 1 of $\S 3$, that $\bar{\delta}$ can be extended to $A$. This completes the proof of the theorem.

\section{REFERENCES}

[1] Albert, A.A: Structure of Algebras, Amer. Math. Soc. Colloquium Publications, Vol. 24 (1939).

[2] Hochschild, G: Restricted Lie algebras and simple associative algebras of characteristic p, Trans. Amer. Math. Soc., Vol. 80 (1955), pp. 135-147.

[ 3 ] Jacobson, N: Abstract derivation and Lie algebras, Trans. Amer. Math. Soc., Vol. 42 (1937), pp. 206-224.

[4] Jacobson, N: Structure of rings, Amer. Math. Soc. Colloquium Publications, Vol. 37 (1956).

Tata Institute of Fundamental Research,

Centre for Advanced Study \& Research in Mathematics,

University of Bombay,

$B O M B A Y$. 